\title{
MODELO DE PREVENCIÓN DE LA VIOLENCIA Y EDUCACIÓN PARA LA PAZ EN ADOLESCENTES ESCOLARES DE UNA INSTITUCIÓN EDUCATIVA EN CUNDINAMARCA
}

\author{
Leydi Migreth Díaz González¹, Andrea Lorena Martínez Rodríguez²
}

\section{Resumen:}

El proceso de la educación para la paz, específicamente desde el campo de la educación, viene desarrollándose desde hace varias décadas, incluso compartiendo momentos clave en el proceso evolutivo de los acercamientos entre diferentes gobiernos y los grupos insurgentes, es decir, los procesos de paz en Colombia han venido permeando las diferentes instancias educativas. El objetivo de la investigación se enfocó en diseñar e implementar un Modelo de Prevención de la Violencia y Educación para la Paz en adolescentes escolares, promoviendo habilidades de autoconocimiento, manejo de las emociones, comunicación empática y resolución de conflicto para una cultura de paz. Para tal fin se siguieron los pasos metodológicos de un proyecto de intervención de caracterización, diseño pedagógico, intervención por medio de talleres, análisis de resultados y evaluación. Se desarrolló así un Modelo de Prevención de la Violencia y Educación para la Paz como iniciativa para evitar la violencia escolar.

Palabras clave: violencia, educación para la paz, resolución de conflictos.

Historial del artículo:

Fecha de recibido: 18-11-2020 - Fecha de aceptado: 12-02-2021

(c) 2021 Fundación Universitaria Juan N. Corpas. FUJNC.

Artículo Open Access bajo la Licencia Creative Commons Atribución-NoComercial-Compartirlgual 4.0 Internacional.

DOI: 10.26752/revistaparadigmassh.v2i2.514

${ }^{1}$ Enfermera, Especialista en Docencia Universitaria, Magistra en Estudios de Paz y Resolución de Conflictos, Docente Universidad Manuela Beltrán. Fundación Universitaria del Área Andina. Autor para correspondencia. Correo electrónico: "Idiaz1115@gmail.com"

2Politóloga, Magistra en Estudios de Paz y Resolución de Conflictos. Correo electrónico: amlore3@gmail.com 


\title{
VIOLENCE PREVENTION AND EDUCATION FOR PEACE MODEL IN SCHOOL ADOLESCENTS OF AN EDUCATIONAL INSTITUTION IN CUNDINAMARCA
}

\begin{abstract}
:
The process of education for peace, specifically from the field of education, has been developing for several decades, even sharing key moments in the evolutionary process of rapprochement between different governments and insurgent's groups that is, the peace in Colombia have been permeating the different educational instances. The objective of the research was focus on the designing and implementing a Model of the Prevention of Violence and Education for Peace in School adolescents, promoting self-knowledge skills, managing emotions, empathic communication, and conflict resolution for a culture of peace. To this end, the methodological steps and intervention project of characterization, pedagogical design, intervention through workshop, analysis of results and evaluation were followed. Thus, a Model for Prevention of Violence and Education for Peace was developed as an initiative to prevent school violence.
\end{abstract}

Keywords: violence, peace education, conflict resolutions. 


\section{Introducción}

El proceso de la educación para la paz, específicamente desde el campo de la educación, viene desarrollándose desde hace varias décadas, incluso compartiendo momentos clave en el proceso evolutivo de los acercamientos entre diferentes gobiernos y los grupos insurgentes, es decir, los procesos de paz en Colombia han venido permeando las diferentes instancias educativas. En ese orden de ideas, "Colombia ingresa a la plataforma epistémica y práctica de las corrientes emergentes de la literatura de paz que, debido a su propio proceso interno, puede dar cuenta de una producción de saberes y aportes nuevos tanto nacionales como internacionales" (Cruz, 2018).

De esta forma, para la primera década del 2000, diferentes instituciones educativas como la Universidad Pedagógica y la Universidad Javeriana y organizaciones como JUSTAPAZ (Centro Cristiano Justicia Paz y Acción No-Violenta) e INDEPAZ, entre otros, comenzaron a publicar textos acerca de proyectos educativos o pedagógicos en relación con la paz. Por otra parte, hay una fracción del trabajo en Pedagogías para la Paz en Colombia que inicia entonces desde el ámbito más formal de la educación en instituciones educativas, bajo la premisa de reducir la violencia en el aula y centrarse en el aprendizaje de valores democráticos y ciudadanos (Adarve, P., González, S., y Guerrero, M. 2018). Es aquí donde cobra interés esta convergencia, en la interrelación del proceso de educación para la paz y la violencia escolar.

Por otro lado, la academia en Colombia históricamente se ha centrado más en estudiar la violencia que la paz. Sin embargo, dentro de los estudios de la paz podemos distinguir tres líneas, a saber: 1. Aquella que surge de los centros internacionales de estudios sobre Colombia; 2. La que se enfoca en los Estudios para la Paz y los movimientos sociales (desarrollados en escenarios locales y en centros de pensamiento e investigación); y 3. Los estudios sobre las negociaciones de Paz en la Habana, Cuba (Cruz, 2018). De allí que cobre cada vez mayor importancia enfatizar en los estudios de paz y sustituirlos por el énfasis en la violencia. Lo anterior es lo deseable y lo que por fortuna está fortaleciéndose cada vez más: una Educación por la paz y para la paz del país.

En la más reciente Encuesta de Clima Escolar y Victimización realizada en 2015, en la ciudad de Bogotá D.C., se registró un alto número de pandillas y actos de violencia que afectan a los estudiantes $y$, en algunas oportunidades, incitan a su participación. En 2016 se presentaron 30.241 presuntos casos que alteraron el clima escolar y la vulneración de derechos en los colegios del Distrito. Las localidades de San Cristóbal, Bosa, Rafael Uribe Uribe y Ciudad Bolívar concentraron $42 \%$ de los presuntos casos de abuso y violencia detectados en el colegio. En Bogotá, los colegios de las localidades de Santa Fe, Rafael Uribe Uribe y Suba, según datos de la Secretaría de Educación Distrital, aún presentan problemas importantes de clima escolar. Para afrontar esta situación, desde el 2016 la entidad desarrolló un plan integral con las secretarías de Cultura, Seguridad y Gobierno con el fin de recuperar estos entornos.

Se determinó, por criterios de acceso, considerando que en la Ciudad de Bogotá se han venido realizando múltiples programas de 
intervención, realizar el presente Modelo de Prevención de la Violencia y Educación para la Pazen el Municipio de Madrid, Cundinamarca, con la metodología cualitativa, aspecto que se describirá más adelante. Con base en el reporte verbal de la psicorientadora Andrea Castelblanco de la Institución Educativa Departamental Serrezuela de Madrid, Cundinamarca, en la observación directa y en el reporte de vecinos del Colegio, se evidencian comportamientos de violencia escolar entre los adolescentes, específicamente en los estudiantes de los grados $6^{\circ}$ y $7^{\circ}$, quienes han mostrado conductas de agresión física y verbal, encuentros fuera del Colegio para resolver a golpes sus conflictos, peleas entre niñas adolescentes con jalones de cabello y arañazos en el rostro, entre otros comportamientos relevantes. Este escenario motivó la estructuración del presente proyecto de intervención, cuyo objetivo general se enfocó en diseñar e implementar un Modelo de Prevención de la Violencia y Educación para la Paz en adolescentes escolares, promoviendo habilidades de autoconocimiento, manejo de las emociones, comunicación empática y resolución de conflicto para una cultura de paz.

\section{Metodología}

Para la estructuración del Modelo de Prevención de la Violencia y Educación para la Paz en Adolescentes Escolares, se adoptaron fundamentos de conceptualización de un modelo pedagógico, con el uso de una metodología cualitativa. Teniendo en cuenta que el propósito del presente proyecto fue entender, en su contexto natural y social, a los adolescentes, el hecho de haber registrado variables cualitativas a través de las conversaciones, la participación en talleres y la observación en dinámicas, se constituyó en una herramienta fundamental para el logro de este.

Para hacer realidad el Modelo de Prevención de la Violencia y Educación para la Paz, se desarrollaron cuatro etapas a saber: 1. Etapa de Caracterización 2. Etapa de Implementación. 3. Etapa de Análisis de Resultados y 4. Etapa de Evaluación, tal como se ilustra en el siguiente esquema: 


\section{Etapa de Caracterización}

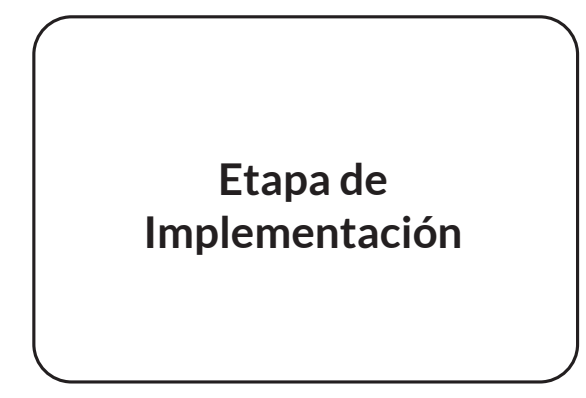

\section{Etapa de Análisis de Resultados}

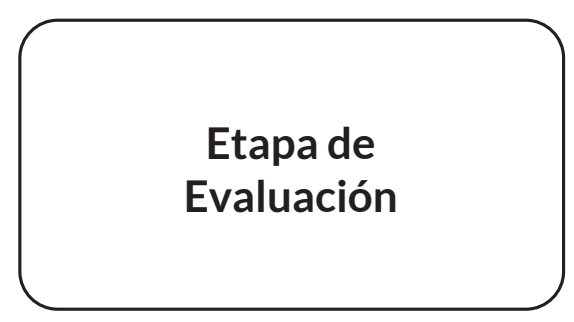

Actividades: Evaluación de resultados por talleres.

Evaluación general.

Recursos: Retroalimentación de los adolescentes escolares participantes.

Análisis sobre evindecias.
Actividades: Aplicación de (4) cuatro talleres.

1. Mi cuerpo: autopercepción de mi cuerpo

2. Emociones/Sentimientos.

3. Comunicación no violencia.

4. Relación con el otro: resolución de conflictos interpersonales en el aula.

Recursos: Espacios físicos: salones y espacios recreativos. Equipos audiovisuales: video beam, computadores, portátiles, amplificadores de sonido.

Materiales didácticos: hojas, papeles de colres, lápices, boligrafos, marcadores, cartulinas.

Actividades: Análisis de resultados por tallers.

Recursos: Análisis de evidencias: ejercicios, dibujos, respuesta ante cuestionario/Observación de desempeño de los participantes 


\section{Etapa de caracterización}

para realizar la etapa de caracterización en el presente proyecto, se procedió primero al proceso de revisión documental, con el propósito de identificar y comprender la conceptualización de categorías de análisis sobre violencia escolar y educación para la paz, apoyado en autores expertos en el tema. Posteriormente se realizaron entrevistas a directivos, coordinadores académicos, a la psicorientadora y a docentes de la institución. Los parámetros que se utilizaron para la aplicación de dichas entrevistas fueron: la estructura y funcionamiento familiar, las condiciones económicas, la dimensión emocional, la dimensión interpersonal y la dimensión social. Además, se realizaron momentos de observación de los adolescentes en su interacción natural durante su estancia en el colegio.

\section{Etapa de implementación}

se realizó el diseño y aplicación de un ciclo de talleres de Construcción de cultura de paz. Luego del proceso de caracterización de la población de estudio y del diseño de un Modelo de Prevención de la Violencia y Educación para la Paz en adolescentes escolares, se procedió a la planeación y aplicación de un ciclo de cuatro talleres. Todos los talleres se desarrollaron con una misma estructura, que comprendió: 1. Diálogo y sensibilización; 2. Conciencia. 3. Reflexión y 4. Compromiso.

\section{Etapa de análisis de resultados}

Este se realizó por cada uno de los cuatro talleres que se aplicaron siguiendo la estructura común a todos fue: 1. Preparación y discusión; 2. Profundización; 3. Interiorización y 4. Responsabilidad. Se utilizaron diferentes recursos apropiados para esta etapa, tal como el análisis de evidencias: ejercicios, dibujos, respuestas ante los diferentes cuestionarios. Así mismo, se utilizó la observación de desempeño de los participantes en cada una de las actividades y dinámicas que dirigieron las talleristas.

\section{Etapa de Evaluación}

Esta se realizó a partir de la evaluación particular porcadaunodeloscuatrotalleres y una evaluación general. Se tomó como técnica de evaluación la retroalimentación de los adolescentes escolares participantes y el análisis sobre evidencias.

\section{Aspectos éticos}

Para la realización de la presente investigación se contó con el consentimiento informado de los padres de los participantes, previa explicación del objetivo del estudio, ventajas y desventajas y toda la información relacionada con el anonimato de los datos y la reciprocidad de los resultados de la investigación para la institución educativa y el grupo de padres. Estos fueron recolectados y archivados por la orientadora de la institución.

\section{Resultados}

En un primer momento se pudo registrar que los adolescentes participantes provienen de familias de estratos 1 y 2 , de zonas rurales y también aledañas al colegio. Los adolescentes que viven en las zonas rurales más lejanas caminan hacia el colegio hasta media hora, otros no desayunan en sus hogares y solo consumen el refrigerio que les aporta el colegio en el descanso en horas de la mañana. ${ }^{3}$

${ }^{3}$ Información suministrada por la psicorientadora de la Institución, Andrea Castelblanco, previa a la realización de los talleres. Adicionalmente se constata con información reportada por un estudiante quien expresó que ese alimento era lo único con lo que podían desayunar. 
Los estudiantes del grado $6^{\circ}$ presentan edades entre los 10 a 13 años, mientras que los del grado $7^{\circ}$ oscilan entre 14 a 17 años. Los estudiantes tienden a agruparse por cercanía de edades y no hay una buena interacción entre hombres y mujeres, es decir, los hombres siempre están con los de su género a excepción de un estudiante de grado sexto quien siempre se encuentra con un grupo de niñas de su curso.

Los estudiantes manifiestan sentimientos de inferioridad y culpabilidad, pues hay compañeros de ellos que los humillan frecuentemente por tener el uniforme roto o por comentarios sobre su aspecto físico, olor y apariencia. Como una forma de defenderse frente a ofensas o agresiones, se han visto obligados a utilizar palos, piedras, cuchillos, y, en casos excepcionales, también armas de fuego que consiguen en sus lugares de residencia.

\section{De los Talleres, hacia una cultura de paz}

Posteriormente en una segunda etapa se aplicaron cada uno de los cuatro talleres programados. 1. Mi cuerpo: autopercepción de mi cuerpo. 2. Emociones / Sentimientos. 3. Comunicación no violenta. 4. Relación con el otro: resolución de conflictos interpersonales en el aula. En general, se aplicaron durante cuatro días en las instalaciones del Instituto Educativo Departamental Serrezuela del Municipio de Madrid, Departamento de Cundinamarca, con una intensidad en promedio de 1 hora cada uno, en las horas de la mañana.

\section{Discusión}

\section{Taller 1: Mi Cuerpo: autopercepción de mi cuerpo.}

Al preguntarles ¿qué es lo que menos les gusta de su cuerpo?, contaron que una de las partes de su cuerpo que menos les gustaba eran los dientes(12estudiantes), nariz(10estudiantes) y su baja estatura (5 estudiantes). Estos datos coinciden con las burlas permanentes que se observaronentre los adolescentes sobre estas características físicas. Así, los adolescentes reportaron escuchar apodos como: "dientón/ dientona", "Narizón/narizona" y/o "enano/ enana, entre otros apelativos, que, dicho por ellos mismos, son considerados formas de agresión hacia las personas. Si bien no se encontró una correlación entre amenazas, apodos y golpes de parte de otros hacia el adolescente y el rechazo hacia alguna parte específica de su cuerpo (y que no es propósito de esta investigación) sí se encontraron casos en los que los adolescentes reportaron baja autoestima y rechazo de partes de su cuerpo, asociados al maltrato físico y verbal de padres de familia y compañeros de clase.

Así, en la relación de la corporalidad y el modo de vivir de los individuos, Maturana (1995) explica cómo un niño al crecer en un contexto de "amor" aprende a amarse y a cuidar su cuerpo, versus, en contextos hostiles y de agresión hacia el cuerpo del individuo, ese niño en formación seguramente, en su modo de vivir, expresará conductas igualmente hostiles.

Frente al interrogante ¿cómo cuido mi cuerpo?, la gran mayoría respondieron que lo cuidan por medio del baño diario, comiendo sano, no exponiéndose a situaciones de peligro, haciendo deporte y ejercicio, entre 
otros. Con respecto a la pregunta ¿he tenido maltrato en mi cuerpo?, varios estudiantes respondieron que se burlan de su aspecto físico, otros manifestaron amenazas de "a la salida nos vemos", otros han recibido golpes de sus compañeros.

En ese orden de ideas, y siendo coherentes con el énfasis hacia la construcción de una cultura de paz, los adolescentes reconocieron la importancia de cuidar su propio cuerpo y el cuerpo del otro como base fundamental del bienestar propio y el bienestar del otro, como el inicio de un proceso de construcción de paz.

\section{Taller 2: Emociones / Sentimientos}

A partir de la participación en múltiples actividades (ver videos, charlas en grupo, dibujo de mural de compromiso de cambio, etc), los adolescentes debieron identificar diferentes emociones y sentimientos, para luego empezar a evaluar sus propias emociones y sentimientos ante situaciones específicas.

Rabia, dolor, tristeza, alegría, enfado, miedo, deseo de venganza, entre otros, fueron el cúmulo de emociones que analizaron los estudiantes durante su participación en el taller. Ante la pregunta ¿cómo suelen expresar sus emociones?, en su mayoría los adolescentes comentaron: burlándose, diciendo groserías, peleando, expresando ira, llanto, gritando y acosando a otros, incluso algunos golpeándose a sí mismos.

Las palabras, a propósito del comentario del adolescente, como los apodos que le ponemos a los otros, Ilevan consigo una carga emocional entre un puente muy indivisible de broma o de burla. Si la persona que recibe el apodo la interpreta como burla, estamos generando un malestar con nuestra expresión, estamos agrediendo intencionalmente o sin intención al otro. De allí que sea importante evaluar la expresión de nuestras emociones en la interacción con los otros, así sean situaciones de aparente broma o juego; lo que puede representar alegría para unos, puede ser tristeza o depresión para el otro.

\section{Taller 3: Comunicación no violenta}

En este taller, los adolescentes tuvieron que analizar múltiples situaciones conflictivas y las diferentes formas de abordarlas. Si bien una agresión recibida por parte de otra persona no genera de forma lógica una sonrisa, como sucedió con varios de los grupos, sí es posible observar las cosas de manera distinta a pensar inmediatamente en agredir al otro.

Otra actividad realizada en el taller fue la exhibicióndeimágenesalosadolescentescuyos contenidos expresaban burla, intimidación, intento de agresión física y exclusión por parte de adolescentes en contextos escolares. Los participantes interpretaban con sus palabras lo que observaban, como un primer paso en el entrenamiento de una comunicación empática -no violenta.

De acuerdo con este taller los participantes analizaron cuál era la reacción natural de las personas ante dichas situaciones (a lo que reportaron un manejo agresivo y violento en defensa de sí mismos) y cómo se podría proceder para superar el problema y no generar más violencia. Las respuestas fueron coherentes con los resultados obtenidos en los talleres anteriores: tranquilizarse (autocontrolarse), dialogar con los atacantes, mejorar su autoestima, comunicar a padres y/o profesores la situación, pedir ayuda a adultos, entre otros. 


\section{Taller 4: Relación con el otro: resolución de conflictos interpersonales en el aula}

En la dinámica se enfatizó sobre el poder de la comunicación no verbal. Sin haber requerido palabra alguna, los adolescentes reportaron lo fácil e inmediato que era identificar lo que estaba expresando el otro con sus cuerpos, movimientos, gestos faciales, movimiento de manos, miradas y posturas. Reconocieron que cada uno de nosotros puede propiciar diálogos positivos o interacciones conflictivas con el otro. De allí que, en este último taller, los adolescentes identificaron factores que facilitan buenas relaciones interpersonales y otros que obstaculizan o generan conflictos.

Las ideas, pensamientos, los apodos, el mal trato, la exclusión, el matoneo, entre otras conductas de violencia escolar, son las fuentes de conflictos permanentes entre los estudiantes. Todos estos tipos de comportamiento hacen parte de lo que se considera intimidación. Chaux et al. (2007) explican cómo la intimidación escolar se presenta cuando algunos estudiantes humillan constantemente a otro/a, se burlan de él/ ella, le ponen apodos ofensivos, lo/a golpean e insultan repetidamente, lo/a excluyen siempre de trabajos en grupo y de fiestas y/o le mandan mensajes ofensivos por Internet y otros medios. Estos comportamientos se evidenciaron en la institución educativa.

Los adolescentes demostraron ser capaces de incorporar nuevas habilidades para resolver conflictos interpersonales. Lo que deduce que el problema no está en ellos, sino que ellos son víctimas de una sociedad injusta, maltratante y desigual. Estos adolescentes son producto de unos contextos violentos de forma permanente que modelan su comportamiento y los condicionan a ser igualmente violentos.

\section{Conclusiones}

El país cuenta con un marco normativo para una Educación para la Paz, por ejemplo, con la Ley 1620 de 2013 ( Sistema Nacional de Convivencia Escolar y formación para el ejercicio de los Derechos Humanos, la Educación para la Sexualidad y la Prevención y Mitigación de la Violencia Escolar) con la cual el Gobierno Nacional del momento reconoció queunodelosretos depaís,estáenlaformación para el ejercicio activo de la ciudadanía y de los Derechos Humanos, a través de una política de promoción y fortalecimiento de la convivencia escolar, enfatizando en las experiencias vividas por los estudiantes, en el desarrollo de su personalidad, en la construcción de sus proyectos de vida y el establecimiento de relaciones positivas con los otros. No obstante, se requiere un mayor esfuerzo para desdibujar escenas como las presenciadas durante la implementación del presente Modelo de Prevención de la Violencia y Educación para la Paz, donde se evidenció cómo un coordinador de disciplina genera violencia hacia los adolescentes.

Para Maturana (1995) es fundamental que los niños y adolescentes crezcan en ambientes sociales sanos, amorosos para generar posteriormente adultos generosos. En el colegio se evidenció que los adolescentes son maltratados verbalmente por docentes y coordinadores, incluso de disciplina. Esto no es coherente con lo que se espera en la construcción de una cultura de paz. Lo que amerita diseñar estrategias que inhiban la generación de esa violencia estructural Galtung (2003), Krauskopf 2006). No es posible consolidar una cultura de paz desde un contexto educativo violento, donde los actores que precisamente deben promover 
una educación para la paz son los principales actores motivadores de conductas violentas.

La educación para la paz es un acto dialógico, al mismo tiempo riguroso, intuitivo, imaginativo y emocional (Cabezudo y Haavelsrud, 2010). Es un proceso que requiere de una racionalidad comunicativa dialógica y los actos de conocer y pensar están directamente ligados entre sí ya que el conocimiento requiere una expresión comunicacional (Morrow y Torres, 1995). "Educar para la paz supone cultivar en los alumnos valores como la justicia, la cooperación, la solidaridad, el desarrollo de la autonomía personal y la toma de decisiones (...)" (Zurbano Díaz, 1998, p.6). Todo este conjunto de valores finalmente genera individuos pacíficos, capaces de ser cooperativos, de establecer vínculos solidarios entre ellos mismos, dando lugar a una cultura de paz, tal como lo expresaron los adolescentes, toda vez que sintieron satisfacción cuando se les reforzaban sus valores y advertían de lo importante que eran para ir construyendo una cultura de paz.

La nueva realidad del país, con el logro del Acuerdo Final para la Terminación del Conflicto Armado y la Construcción de una Paz Estable y Duradera, (entre el anterior Gobierno Nacional y las FARC-EP), invita a transformar los sentimientos, hábitos y formas de relacionamiento afianzados por la guerra. (Arboleda et al., 2017). Este nuevo escenario motiva transformaciones políticas y económicas de los territorios más impactados por el conflicto armado, la reconstrucción del tejido social, con la implementación de garantías de no repetición, empezando por los compromisos que debe cumplir el Estado.

Se requiere fortalecer esas emociones evaluadoras de las que habla Nussbaum, como también de una educación emocional, que se distancia de los planteamientos de inteligencia emocional de Goleman (1999), para ir reconstruyendo una paz desde lo individual, desde lo micro, desde lo local. Así, con inteligencia emocional es posible una educación y cultura de paz que sea capaz de reducir y prevenir la violencia en el campo educativo. Es importante además que se desarrollen en los niños y adolescentes competencias ciudadanas, es decir, capacidades emocionales, cognitivas y comunicativas que, integradas con conocimientos y disposiciones, hagan posible que las personas puedan actuar de manera constructiva en la sociedad (Chaux, Lleras y Velásquez, 2004). 


\section{Referencias bibliográficas}

1. Adarve, P., González, S., y Guerrero, M. (2018). Pedagogías para la Paz en Colombia: un primer acercamiento. Revista Ciudad Paz-ando, 11(2), pp. xx-xx. doi: https://doi. org/10.14483/2422278X.13177

2. Arboleda,Z,Herrera, M.\&Prada R,M. (2017)¿Quées educaryformar parala pazycómo hacerlo? Educación y Pedagogía para la Paz Material para la práctica. Oficina del Alto comisionado para la paz. Acción CaPaz: Estrategia de Capacidades para la Paz y la Convivencia. Bogotá: OACP.

3. Cabezudo, A. y Haavelsrud, M. (2010). Repensar la educación para la cultura de paz. PROSPECTIVA. Revista De Trabajo Social E IntervencióN Social (15): 71-104.

4. Chaux, E., Bustamante, A., Castellanos, M., Jiménez, M. y Mejía, M.I. (2007). Kit Papaz para el manejo de la intimidación escolar. Bogotá: Universidad de los Andes.

5. Chaux, E; Lleras, J. y Velásquez, A. (2004). Competencias ciudadanas: de los estándares al aula: una propuesta de integración a las áreas académicas. Bogotá: Ediciones Uniandes.

6. Cruz, J.D. (2018). Los estudios de paz latinoamericanos en la encrucijada. Revista CoPaLa. 3(5): 9-21.

7. Galtung, J. (2003). Violencia Cultural. Documentos de trabajo Gernika Gogoratuz 14.

8. Goleman, D. (1999) La práctica de la Inteligencia emocional. Barcelona: Kairos.

9. Krauskopf, D. (2006). Estado del arte de los programas de prevención de la violencia en ámbitos escolares. Lima: Pan American Health Organization. Cooperación Técnica Alemana-GTZ.

10. Ley 1620 Por la cual se crea el Sistema Nacional de Convivencia Escolar y Formación para el Ejercicio de los Derechos Humanos, la Educación para la Sexualidad y la Prevención y Mitigación de la Violencia Escolar. (2013). Diario Oficial No. 48.733. 15 de marzo de 2013

11. Maturana, H. (1995) "Biología y Violencia”. En Violencia en sus Distintos Ámbitos de Expresión (pp.71-91). Santiago de Chile: Dolmen Ediciones, 1995.

12. Morrow, R. A. y C. A. Torres (1995). Social theory and education: a critique of theories of social and cultural reproduction. Albany: State University of New York Press.

13. Zurbano Díaz, J. (1998). Bases para una educación para la paz y la convivencia. Pamplona: Gráficas Ona. 